\title{
Papilledema: epidemiology, etiology, and clinical management
}

This article was published in the following Dove Press journal:

Eye and Brain

17 August 2015

Number of times this article has been viewed

\author{
Mohammed Rigi' \\ Sumayya J Almarzouqi ${ }^{2}$ \\ Michael L Morgan ${ }^{2}$ \\ Andrew G Lee ${ }^{2-4}$ \\ 'Robert Cizik Eye Clinic, University \\ of Texas, ${ }^{2}$ Department of \\ Ophthalmology, Houston Methodist \\ Hospital, Blanton Eye Institute, \\ ${ }^{3}$ Baylor College of Medicine, \\ ${ }^{4}$ Departments of Ophthalmology, \\ Neurology, and Neurosurgery, Weill \\ Cornell Medical College, Houston, \\ UTMB Galveston, UT MD Anderson \\ Cancer Center, Houston, TX, \\ The University of lowa Hospitals and \\ Clinics, lowa City, IA, USA
}

Correspondence: Andrew G Lee Department of Ophthalmology, Houston Methodist Hospital, Blanton Eye Institute, Scurlock 450, 6560 Fannin Street,

Houston, TX 77030, USA

$\mathrm{Tel}+|7| 344 \mid 8843$

Fax +I 7I3 7931636

Email aglee@houstonmethodist.org
Abstract: Papilledema is optic disc swelling due to high intracranial pressure. Possible conditions causing high intracranial pressure and papilledema include intracerebral mass lesions, cerebral hemorrhage, head trauma, meningitis, hydrocephalus, spinal cord lesions, impairment of cerebral sinus drainage, anomalies of the cranium, and idiopathic intracranial hypertension (IIH). Irrespective of the cause, visual loss is the feared morbidity of papilledema, and the main mechanism of optic nerve damage is intraneuronal ischemia secondary to axoplasmic flow stasis. Treatment is directed at correcting the underlying cause. In cases where there is no other identifiable cause for intracranial hypertension (ie, IIH) the available options include both medical and surgical modalities. Weight loss and diuretics remain the mainstays for treatment of $\mathrm{IIH}$, and surgery is typically reserved for patients who fail, are intolerant to, or non-compliant with maximum medical therapy.

Keywords: papilledema, intracranial hypertension, idiopathic intracranial hypertension, epidemiology, papilledema management, papilledema etiology, acetazolamide, optic nerve sheath fenestration, ventriculoperitoneal shunt, lumboperitoneal shunt, venous sinus stenting

\section{Introduction}

The term "papilledema" for the purposes of this review is defined as optic disc edema secondary to high intracranial pressure (ICP). Other terms are used to refer to disc edema from other etiologies. By definition, papilledema cannot exist in the absence of high ICP, but high ICP can occur in the absence of papilledema. Papilledema is frequently bilateral and symmetric, but may be asymmetric or unilateral. The etiology for the high ICP or intracranial hypertension (IH) may be known (eg, brain tumor, meningitis, cerebral venous sinus [CVS] thrombosis) or may be unknown (ie, idiopathic). ${ }^{1}$ In this review article, we discuss the epidemiology, etiology, and clinical management of papilledema.

\section{Epidemiology}

Papilledema from various causes of IH may develop at any age, in either sex, and in any racial or ethnic group. Although high ICP can occur in infants and very young children, open fontanels may mitigate the development of papilledema in these patients despite $\mathrm{IH}^{2}$

In contrast, idiopathic intracranial hypertension (IIH) predominantly affects obese women of childbearing age (Table 1). In the USA, the annual incidence per 100,000 persons has been estimated to be 0.9 in the general population and 3.5 in females $15-44$ years of age..$^{3,4}$ Durcan et al estimated that the incidence of IIH is up to 
Table I Incidence of idiopathic intracranial hypertension

\begin{tabular}{llll}
\hline Country & Incidence in general population & Incidence in women (childbearing age) & $\begin{array}{l}\text { Incidence in obese women } \\
\text { (childbearing age) }\end{array}$ \\
\hline USA & $0.9 / 100,000$ & $3.5 / 100,00$ & $13 / 100,000>10 \%$ ideal body weight \\
& & & $19.3 / 100,000 \geq 20 \%$ ideal body weight \\
UK & $1.56 / 100,000$ & $2.86 / 100,000$ & $11.9 / 100,000$ \\
Middle East & $2.02-2.2 / 100,000$ & & \\
\hline
\end{tabular}

13 per 100,000 in obese women aged $20-44$ years of age who were $\geq 10 \%$ overweight and up to 19.3 per 100,000 if $\geq 20 \%$ over ideal weight. ${ }^{3}$ The incidence of IIH varies from country to country, probably related to the prevalence of obesity. The incidence was 1.56/100,000 persons/year, 2.86/100,000 in women, and 11.9/100,000 in obese women in the Sheffield UK study. ${ }^{5}$ The reported annual incidence of IIH in Middle Eastern countries has been estimated at 2.02-2.2/100,000 in the general population. ${ }^{6}$

Although less common, IIH can also occur in children, men, non-obese patients, and the elderly. However, these atypical IIH patients should undergo a more aggressive evaluation for underlying etiologies other than IIH.

\section{Pathogenesis of papilledema}

The pressure-volume relationship between ICP, volume of cerebrospinal fluid (CSF), blood, brain tissue, and cerebral perfusion pressure is known as the Monro-Kellie doctrine. Because the total volume inside the cranium of blood, CSF, and brain is fixed, any increase in volume of one of the cranial constituents must be compensated for by a decrease in volume of the others or high ICP will result. A spaceoccupying lesion often causes $\mathrm{IH}$, which frequently leads to papilledema. $^{7}$

Thus, high ICP may occur by any one or combination of the following mechanisms: an increase in the total amount of intracranial tissue by a space-occupying lesion (eg, brain tumor); an increase in intracranial tissue volume by focal or diffuse cerebral edema; an increase in production of CSF; a decrease in total available volume within the cranial vault by thickening of the skull; a decrease in the outflow of CSF within the ventricular system (eg, obstructive or non-communicating hydrocephalus) or within the arachnoid granulations (eg, meningitis, subarachnoid hemorrhage [SAH]); and a decrease in the absorption of CSF from intracranial or extracranial obstruction or compromise of venous outflow (eg, venous sinus thrombosis). Another potential mechanism of high ICP in IIH is high intraabdominal pressure, which may increase pleural pressure and cardiac filling pressure, leading to increased intracranial venous pressure and high ICP. ${ }^{8}$ Table 2 lists a number of causes for high ICP.

\section{Mechanism and pattern of visual loss}

The main mechanism of visual loss is likely due to axoplasmic flow stasis. High ICP produces a rise in CSF pressure surrounding the optic nerves, which disturbs the normal gradient between intraocular pressure and retrolaminar pressure, leading to high tissue pressure within the nerves. The increased tissue pressure within the nerves interrupts the metabolic processes that mediate axoplasmic flow. ${ }^{9-11}$

In the acute stage, papilledema leads to enlargement of the blind spot, which is the most common and often the only visual field change. ${ }^{12}$ With protracted and severe papilledema, nerve fiber layer visual field defects occur. The typical visual field defects found in papilledema are related to nerve fiber bundle damage at the level of the optic disk. ${ }^{13}$ The papillomacular bundle and thus central

Table 2 Causes of increased intracranial pressure

Space-occupying lesions

- Intracranial mass

- Abscess

- Hemorrhage

- Arteriovenous malformation

Focal or diffuse cerebral edema

- Trauma

- Toxic

- Anoxia

Reduction in size of the cranial vault

- Craniosynostosis

- Thickening of skull

Blockage of CSF flow

- Non-communicating hydrocephalus

Reduction in CSF reabsorption

- Communicating hydrocephalus

- Meningitis

- Elevated cerebral venous sinus pressure

- Elevated CSF protein

Increased CSF production

Idiopathic intracranial hypertension

Abbreviation: CSF, cerebrospinal fluid. 
visual acuity appears spared until later stages of the disease. $^{14}$

\section{Etiology of papilledema Mass lesions}

An increase in intracerebral volume from a mass lesion may produce high ICP leading to papilledema, but compensatory mechanisms may preclude the development of papilledema in chronic cases. In one series, papilledema was found in only $28 \%$ of patients aged $0-90$ years with a history of brain tumor presenting to an emergency department, but the sensitivity and reliability of detection of papilledema in this setting may be low. ${ }^{15}$ In contrast, several larger neurosurgical series found papilledema in up to $60 \%-80 \%$ of patients with cerebral tumors. $^{16}$

Infratentorial mass lesions, which may obstruct the ventricular outflow at the relatively narrow Sylvian aqueduct are more likely to produce papilledema than supratentorial mass lesions. Brain tumors in children are more commonly located in the posterior fossa, and thus present more frequently with papilledema. $^{17}$

\section{Cerebral hemorrhage}

$\mathrm{SAH}$, acute subdural hematoma (SDH), and intraparenchymal hemorrhage (IPH) may all be associated with papilledema, and may develop within hours of the hemorrhage. Papilledema due to SAH or IPH occurs in only a minority of patients, despite the presence of high ICP. In one series of patients with ruptured aneurysms, papilledema was found in only $16 \%$ of patients despite $\mathrm{SAH} .{ }^{18}$ The presence of papilledema in $\mathrm{SAH}$ was not related to sex, age, or aneurysm site. Interestingly, in this series of seven cases, the papilledema was unilateral, and was ipsilateral to the lesion in six cases. It is believed that SAH produces papilledema either by blocking CSF outflow within the ventricular system or by blocking CSF absorption at the arachnoid granulations. Likewise, the incidence of papilledema in patients with aneurysmal SAH has been reported by other investigators as 10\%-24\%.

Papilledema also occurs in patients with both acute and chronic SDH, but is more commonly observed in the acute phase. In contrast, in patients with epidural hematoma, the papilledema may develop many weeks after the injury, particularly when the hematoma is located at the vertex causing compression of the superior sagittal sinus. ${ }^{19}$

\section{Trauma}

In addition to traumatic intracranial hemorrhage (eg, $\mathrm{SAH}$, $\mathrm{SDH}$, epidural hematoma, IPH), trauma can produce high
ICP and papilledema via other mechanisms. In one study of patients with acute head injury, the presence of papilledema had little correlation with the degree of high ICP and it was found only in $3.5 \%$ of patients. ${ }^{20}$ Thus, the absence of papilledema does not necessarily rule out high ICP in these patients. In this study, papilledema was more common in patients with SDH. Papilledema that develops in patients after head trauma is usually described as mild (but is quite variable) and may develop immediately, occur several days after the injury, or up to 2 weeks later. The postulated mechanism for the immediate development of papilledema is a sudden and severe but transient increase in ICP, whereas sustained but mild to moderately high ICP accounts for papilledema that appears during the 1st week after injury. Papilledema during the 2nd week or later may result from impaired CSF absorption and consequent communicating hydrocephalus or delayed focal or diffuse cerebral edema.

\section{Meningitis}

Meningitis may cause high ICP by producing severe secondary cerebral edema, obstructive hydrocephalus, or impaired CSF absorption by inflammation at the level of the arachnoid granulations. The reported frequency of papilledema with meningitis is fairly small, and the papilledema in these cases tends to be mild and transient but is highly variable. The attribution of findings to papilledema alone in meningitis is also complicated by the presence of alternative mechanisms for the optic disc edema other than high ICP, including direct infectious, secondary inflammatory, or infiltrative mechanisms (eg, cryptococcal meningitis). In one study of 2,178 cases of meningitis, only $2.5 \%$ of patients had papilledema, ${ }^{21}$ and other larger older series of syphilitic meningitis showed a similar incidence of papilledema. ${ }^{22}$ In cases of bacterial meningitis, papilledema was more likely to occur in patients with tuberculous meningitis, and was observed in up to $25 \%$ of cases. ${ }^{21}$ Papilledema due to viral or other causes of aseptic meningitis is much less common and was observed in only $2 \%$ of patients in one series. ${ }^{23} \mathrm{~A}$ retrospective review of 100 patients with a definite or probable diagnosis of viral meningitis, encephalitis, or meningoencephalitis reported only six patients with optic disc edema, two of whom had high ICP. In the other four patients, the CSF was under normal pressure, but CSF pleocytosis was present, suggesting that the disc edema was caused by inflammation rather than high $\mathrm{ICP}^{24}$

\section{Hydrocephalus}

Obstructive (non-communicating) hydrocephalus results from compression of the ventricular system or its associated 
foramina, and is another cause of high ICP with resultant papilledema. Some common causes of hydrocephalus include neoplasms, intraventricular or SAH, meningitis, and congenital aqueductal stenosis.

\section{Spinal cord lesions}

Spinal canal tumor is an unusual cause of high ICP and can result in the development of papilledema. Most of these spinal tumors are intradural, but extradural spinal tumors can also cause high ICP. ${ }^{25,26}$ In tumors involving the upper cervical region, the high ICP is thought to be upward swelling of the tumor with compression of the cerebellum and obstruction of CSF flow through the foramen magnum. Over $50 \%$ of spinal cord lesions associated with papilledema are ependymomas or neurofibromas, which are usually in the thoracic and lumbar regions, and so this mechanism alone is unlikely to be the sole cause of the high ICP. ${ }^{27}$

The cause of high ICP and papilledema in these cases is more likely due to impaired CSF absorption resulting from blockage of the arachnoid granulations by increased CSF protein produced by these and other tumors. A similar mechanism might be at work causing papilledema in the Guillain-Barré syndrome. ${ }^{28}$ In other cases, recurrent SAH, which occurs commonly as a result of bleeding from the surface of ependymomas, may also cause impairment of CSF absorption from blockage of the arachnoid villi by blood or blood products. ${ }^{27}$

Papilledema may occasionally be present in patients with other non-neoplastic spinal cord pathology, presumably by similar mechanisms. It has been reported in a patient with a herniated thoracic disc, resolving postoperatively. The authors postulated that the papilledema in this patient was caused either by chronic epidural venous congestion from pressure of the extradural herniated disc producing a partial subarachnoid block, or by an associated aseptic meningitis and an elevated CSF protein. ${ }^{29}$ Other mechanisms include loss of the elastic reservoir compensatory mechanisms in the spinal cord due to tumor.

\section{Idiopathic intracranial hypertension}

IIH is also known as primary pseudotumor cerebri. IIH is typically defined by exclusion using specific diagnostic criteria (eg, modified Dandy criteria). These criteria include: signs and symptoms due only to high ICP (eg, headache, pulse-synchronous tinnitus, papilledema, and diplopia due to a non-localizing sixth nerve palsy); normal neuroimaging (eg, typically magnetic resonance imaging preferably with and without contrast and magnetic resonance venogram); high ICP (typically greater than 25 $\mathrm{cm}$ of water measured in the left lateral decubitus position) but normal CSF composition; and no alternative underlying etiology for the findings. ${ }^{30}$ IIH typically affects obese women of childbearing age, but it may be seen in patients of any age, in either sex, and without obesity. ${ }^{31}$ Risk factors for secondary $\mathrm{IH}$ are the use of exogenous substances such as lithium, hormones (eg, growth hormone, thyroid replacement), vitamin A analogs (eg, retinoids), antibiotics (eg, nitrofurantoin, nalidixic acid, and tetracyclines, but especially minocycline) and intake or more likely withdrawal of corticosteroids. ${ }^{32}$

The diagnosis of IIH is one of exclusion, and the modified Dandy criteria, shown in Table 3, are generally used to make the diagnosis. The disorder is not necessarily benign, although it was once called "benign intracranial hypertension", because patients may suffer significant vision loss related to papilledema and many patients experience moderate to severe and/or intractable headaches. A case-control study showed that higher levels of weight gain and body mass index were associated with a greater risk of IIH. ${ }^{33}$ Recent moderate weight gain (5\%-15\% of body weight) in obese and non-obese patients also increases the risk of IIH. ${ }^{34} \mathrm{IIH}$ patients typically present with symptoms and signs of high ICP. Headache is the most commonly reported presenting symptom, occurring in more than $90 \%$ of cases in most studies. ${ }^{4,35,36} \mathrm{IIH}$ patients often suffer from daily headache, and they may be wakened by headache. ${ }^{36}$ Other symptoms may include transient visual obscurations, blurred vision, an enlarged blind spot or other visual field defects, binocular diplopia (due to non-localizing sixth nerve palsy), and pulse-synchronous tinnitus. Vision loss is usually due to papilledema and secondary optic neuropathy, implying that patients without papilledema are not at risk for visual loss. A large majority of patients with IIH have papilledema, but IIH without papilledema has been reported. The papilledema is usually bilateral and symmetric, but can be asymmetric or unilateral in about $10 \%$ of patients. $^{37}$

Table 3 Modified Dandy criteria

Symptoms, if present, and signs representing increased ICP or papilledema

Documented high ICP measured in the lateral decubitus position Normal CSF composition

Normal MRI or contrast-enhanced CT for typical patient and MRI and MRV for all others

No other cause of increased ICP

Abbreviations: ICP, intracranial pressure; MRI, magnetic resonance imaging; $C T$, computed tomography; MRV, magnetic resonance venography; CSF, cerebrospinal fluid. 


\section{Obstruction or impairment of intracranial venous drainage}

Obstruction (eg, thrombosis) of CVS drainage may result in high ICP and papilledema without enlargement of the ventricles and with otherwise normal CSF. Compression or thrombosis of the CVS is the cause of obstruction, with the superior sagittal and transverse (lateral) sinuses most often affected. In cases of septic thrombosis of the CVS, papilledema tends to occur early and to be bilateral and symmetric. ${ }^{38}$ Aseptic thrombosis may occur in CVS of both adults and children, with the superior sagittal sinus most frequently affected. ${ }^{39}$ These patients may have an underlying coagulopathy (eg, Factor V Leiden mutation, prothrombin gene 20210GA transition, activated protein $\mathrm{C}$ resistance, protein C, $\mathrm{S}$, or antithrombin III deficiency, anticardiolipin antibodies, or hyperviscosity syndromes), systemic conditions (eg, renal disease, pregnancy, and cancer), predisposing medication use (oral contraceptives), or systemic inflammatory or infectious diseases (eg, systemic lupus erythematosus, Behçet disease, trichinosis, or sarcoidosis).

Dural arteriovenous fistula has been reported as a rare complication of CVS thrombosis, which may result in recurrence of papilledema. The estimated fistula incidence rate of $1 \%-3 \%$ is based on cohort studies without systemic angiographic follow-up. ${ }^{40}$

\section{Anomalies of the cranium}

The intracranial vault may be smaller in certain types of craniosynostoses. Up to $12 \%-15 \%$ of patients with premature synostosis of the cranial sutures may eventually develop papilledema. ${ }^{41}$ The incidence of papilledema increases up to $40 \%$ in patients with specific craniofacial dysostosis (eg, Crouzon syndrome) or acrocephalosyndactyly (eg, Apert syndrome). In the older literature, up to $50 \%$ of patients with craniosynostosis and papilledema eventually developed severe reduction of vision or blindness in both eyes. In one study of 31 patients with post-papilledema optic atrophy, $52 \%$ had visual acuity of $20 / 60$ or better in both eyes, $29 \%$ had acuity of 20/60 or better in one eye, and only $19 \%$ had visual acuity less than 20/60 in both eyes. Papilledema in craniosynostosis usually develops before the age of 10 years. ${ }^{41}$ Patients with smaller posterior fossa volume and the Chiari malformation may also present with secondary IH and papilledema.

\section{Treatment}

High ICP due to a space-occupying mass, Chiari malformation, or hydrocephalus is typically treated surgically. ${ }^{42,43}$ In the absence of a surgical lesion, patients should be screened for secondary causes of IH and treated accordingly. Medications causing IH should be discontinued if possible. ${ }^{32}$ Management of CVS thrombosis include treatment of the underlying condition and prevention or treatment of its complications, as well as anticoagulation therapy. ${ }^{40}$ In cases where there is no identifiable cause for IH (ie, IIH) the available options include both medical and surgical modalities. A selected summary of clinical management approaches is listed in Table 4. Patients with minimal papilledema, no visual loss, and no other symptoms can be treated conservatively and monitored frequently for development of symptoms or vision loss. The visual field defects may not be recognized by patients, so they should be monitored for visual field defects by formal perimetry. Symptomatic headache treatment can be initiated when appropriate. The main goals of treatment are alleviation of symptoms and preservation of vision. Conservative management options for IIH include weight loss and diet, carbonic anhydrase inhibitors (typically acetazolamide), loop diuretics, and topiramate. Surgery is generally reserved for those patients who fail, are non-compliant with, or are

Table 4 Summary of clinical management considerations

\begin{tabular}{ll}
\hline Etiology of papilledema & Treatment considerations \\
Space-occupying mass, Chiari malformation, or hydrocephalus & Consider specific surgical therapy \\
Secondary causes & Direct treatment to the underlying cause $(s)$ \\
Cerebral venous sinus thrombosis & Treat the underlying cause \\
& Consider adding anticoagulation \\
Potential contributing factors & Eliminate contributing factors \\
IIH with minimal signs or symptoms, and no visual loss & Diet, weight loss \\
& Monitor visual loss by formal perimetry \\
IIH with symptoms and visual loss & Diet, weight loss, diuretics \\
& Monitor closely \\
IIH with failed maximal medical therapy & Consider surgical options \\
IIH with acute fulminant papilledema and visual loss & Consider urgent surgical intervention \\
& Use temporizing measures such as serial lumbar punctures or lumbar drain \\
& to halt visual loss while definitive surgical treatment is awaited
\end{tabular}

Abbreviation: $\mathrm{IH}$, idiopathic intracranial hypertension. 
intolerant of maximal medical therapy. However, patients with acute fulminant papilledema and visual loss usually require urgent surgical intervention to prevent further irreversible visual loss. ${ }^{44}$ The choice between optic nerve sheath fenestration (ONSF) and neurosurgical shunting procedures depends on local surgical expertise, institutional experience and resources, as well as the timing and severity of the patient's symptoms and signs. A lumbar drain during the acute hospitalization might be useful as a temporizing measure prior to definitive surgical intervention in cases of fulminant IIH.

\section{Medical treatment}

The combination of pharmacotherapy, weight loss, and diet is the mainstay of medical treatment of IIH. Weight loss, when it can be achieved, is probably the most effective long-term therapy. It is important to emphasize that only a modest amount of weight loss (5\%-10\% of total body weight) is usually required for improvement in symptoms and signs. ${ }^{45}$ Sinclair et al reported a reduction in CSF pressure in IIH patients treated with a 425 calorie per day diet for 3 months. ${ }^{46}$ These authors demonstrated that weight loss effectively reduces not only headaches but also ICP. Bariatric surgery may be beneficial to morbidly obese IIH patients whose weight loss attempts have been unsuccessful. ${ }^{47}$ Given the significant morbidity associated with obstructive sleep apnea, especially in obese individuals, and the possibility of a link between IIH and obstructive sleep apnea, ${ }^{48-50} \mathrm{IIH}$ patients should be screened for obstructive sleep apnea $^{51}$ and treated appropriately. Evaluation and treatment of other potential contributing factors (eg, medication use and anemia) should be also considered.

\section{Diuretics}

Acetazolamide, a carbonic anhydrase inhibitor, combined with a low sodium weight reduction diet is a popular firstline combination in IIH patients with mild to moderate vision loss. The recently completed IIH treatment trial was a multicenter, randomized, double-blind, placebo-controlled study which concluded that weight reduction coupled with acetazolamide is a safe and effective treatment for IIH. ${ }^{52}$ The combination of acetazolamide and a low sodium weight reduction diet, compared with diet alone, resulted in a statistically significant, albeit small $(0.71 \mathrm{~dB})$, mean improvement of the Humphrey visual field.

We typically begin therapy in patients with $500 \mathrm{mg}$ of acetazolamide twice daily if there is no contraindication. Other authors have recommended an initial lower dose of $500 \mathrm{mg}$ once daily with more rapid advancement to the
$500 \mathrm{mg}$ twice-daily dosage to improve acclimation to side effects and compliance. We generally gradually titrate the dose of acetazolamide as indicated to maximally tolerated doses of up to $2 \mathrm{~g}$ or more per day. Higher doses (up to $4 \mathrm{~g}$ a day) may be needed but generally are not tolerated well. If patients cannot tolerate, are noncompliant with, or fail acetazolamide therapy then we usually consider a second-line alternative or adjunctive furosemide at a dose of $20 \mathrm{mg}$ twice daily and titrating the dose to as much as $40 \mathrm{mg}$, three times daily if needed. Furosemide is a weak carbonic anhydrase inhibitor and chloride reuptake blocker and has been used to treat $\mathrm{IIH} .{ }^{53}$ It appears to be effective in some patients, including those patients who fail acetazolamide, and we use it as our second-line agent after acetazolamide. Unlike acetazolamide, furosemide for IIH has not been studied in a large, randomized controlled manner. Weaker diuretics, including thiazides, digoxin, and glycerol, do not appear to be as effective, but have shown anecdotal success in some patients in the literature. However, it should be noted that there are currently no results from controlled prospective trials assessing and comparing the efficacy of these alternative diuretics used for the treatment of IIH.

\section{Topiramate}

Topiramate has also been used for IIH, and treats primary headache disorders. In one small randomized treatment trial, topiramate appeared to have similar efficacy to acetazolamide for treatment of mild to moderate $\mathrm{IIH} .{ }^{54}$ However, this study lacked a placebo control group, and patients were alternately assigned to the treatments. Topiramate often causes some weight loss as a side effect, which might have an added advantage over other agents used for prevention of headache in IIH. Neurocognitive side effects may limit the use of topiramate in $\mathrm{IIH}$, and is not our first-line agent.

\section{Corticosteroids}

Steroids were once used commonly to treat $\mathrm{IH},{ }^{55,56}$ but their long-term use to lower ICP is not recommended due to the risk of rebound $\mathrm{IH}$ on withdrawal. ${ }^{57,58}$ Further, the unfavorable long-term side effects of steroids such as weight gain in these already obese patients could work against the overall goal of weight reduction in IIH. High-dose intravenous steroids can, however, be utilized as a temporizing measure to treat fulminant IIH patients with acute and severe visual loss until more definitive surgical intervention is completed.

\section{Serial lumbar punctures}

Repeated lumbar punctures to treat IH have also been used $^{55}$ but are not widely recommended because the lumbar 
puncture only transiently reduces CSF pressure as CSF volume reforms shortly after the procedure. ${ }^{59}$ Serial lumbar punctures are also technically challenging and poorly tolerated by patients. Nevertheless, serial lumbar punctures might be considered in the patient who is pregnant or not suitable for medical therapy (eg, cryptococcal meningitis), but who need a repeated short-term temporizing measure to reduce CSF pressure while waiting for a definitive surgical procedure or medical improvement. A temporary lumbar drain can also be used as a bridge to definitive surgical treatment in patients with acute and severe visual loss (eg, acute, fulminant IIH).

\section{Surgical treatment}

There have been no randomized controlled trials to assess the surgical treatment of $\mathrm{IIH}$, and the current available data on the efficacy and complications of surgical intervention originate from observational and retrospective studies. Three recent studies have reviewed the currently available literature on the efficacy and complications of surgical treatment modalities in IIH. ${ }^{60-62}$ Many authors have concluded that there is insufficient evidence to recommend or reject any one surgical treatment modality for IIH. The results of a pooled analysis in one of the reviews showed an overall similar improvement in visual outcomes across treatment modalities and a modest improvement in headache following a CSF diversion procedure and endovascular stent placement. ${ }^{60}$ Further, they found that CSF shunting was associated with a higher rate of periprocedural complications when compared with other interventions.

In general, patients with acute fulminant $\mathrm{IIH},{ }^{44}$ severe vision loss at presentation or progressive vision loss despite maximum medical management, require surgical intervention. ${ }^{63}$ The surgical options include ONSF, CSF diversion (eg, lumboperitoneal shunt [LPS] or ventriculoperitoneal shunt [VPS]), and venous sinus stenting. The intervention of choice depends, in part on local resources, and the biases of the therapeutic team. The therapeutic focus following surgical intervention should be to treat the underlying $\mathrm{IIH}$, mainly by encouraging and facilitating weight loss.

\section{Shunting procedures}

VPS and LPS are CSF pressure-lowering procedures that can be effective in selected patients with IIH who fail maximum medical therapy ${ }^{64-67}$ Recently, Sinclair et al conducted the largest retrospective review of IIH patients treated with shunts (LPS in 49 patients versus VPS in four) ${ }^{68}$ Although this study failed to show an improvement in headaches (19\%) after CSF diversion, improvement of papilledema was significant (44\%) at 24 months. These authors recommend that headaches should not be the sole indication for shunting due to the high rate of shunt complications, shunt revisions, and persistent post-shunt headaches. Shunting procedures could still be considered when both intractable headaches and deterioration of vision despite maximal medical treatment require further management, ${ }^{65}$ and meta-analyses of the published literature seem to support this approach. ${ }^{60,61,69}$

Ventricular shunts are technically more challenging. There are risks involved with placing the proximal catheter in the often small ventricles of IIH patients. ${ }^{70}$ However, VPS seems to have lower the risk of shunt obstruction or shunt revision compared with LPS. ${ }^{60,71-73}$ The number of true revisions could be distorted by the fact that far fewer VPS in general are performed than LPS. Shunting procedures have significant morbidity and some mortality $(0.9 \%$ for VPS and $0.3 \%$ for LPS $)^{67}$ and may be complicated by failure from shunt obstruction, breakage, infection, or rarely sudden severe vision loss. ${ }^{74} \mathrm{CSF}$ shunting procedures seem to have a higher rate of periprocedural complication compared with other interventions, and no significant differences in outcomes have been revealed between LPS and VPS. ${ }^{60}$ One retrospective study found that the patients' characteristics, such as severe or fulminant CSF pressure or poor manometric response to repeated lumber taps, predicted higher success in LPS. ${ }^{75}$ Prospective studies are needed to compare the efficacy of VPS versus LPS. Newer techniques such as stereotactic VPS placement have been introduced, which may help reduce the complications of shunt placement in the future. ${ }^{76}$ Because of the mortality risk, high rates of shunt failure with need for revision $(41 \%-63 \%),{ }^{77}$ the wide range in symptomatic improvement, and the disturbingly high incidence of acquired Chiari I defects and syringomyelia, it is our bias that these shunting procedures should not be used as the first-line or primary treatment for visual loss from papilledema if ONSF is a readily available option. ${ }^{66,67}$ In our institution, for those who require shunting, we favor stereotactically placed programmable valve VPS over LPS for patients with symptomatic IIH who have failed conservative and maximal medical treatment for headache and visual loss due to papilledema. Ultimately, the decision for surgical treatment in IIH is largely dependent on locally available neurosurgical expertise for shunting and oculoplastic/orbit surgical expertise for ONSF. Thus, clinicians have to make an individual judgment based on the surgical treatments available in their community and the needs and preferences of the individual patient. 


\section{Optic nerve sheath fenestration}

ONSF is an effective method for stabilizing visual loss and protecting the optic nerve from further damage in many, but not all, patients with IIH. ${ }^{78-83}$ Keltner proposed that the mechanism of action of ONSF is local decompression of the subarachnoid space. ${ }^{82}$ In addition to orbital drainage, ${ }^{84}$ the long-term mechanism of ONSF remains controversial, but may involve closure by scarring of the subarachnoid space in the retrolaminar optic nerve or continuous function of the fistula. ${ }^{85}$

Complications can arise from direct orbital and intraocular-related effects, ranging from $5 \%$ to $45 \%$ in most studies. ${ }^{86}$ Rarely, however, loss of vision (1\%-2\%) can occur in the perioperative period. ONSF is generally safe, and no mortalities have been reported in the literature. The data from the literature suggest that the complication rate might depend on not only the amount of the stretch placed on the optic nerve but also on ONSF surgical techniques. ${ }^{62}$

The results of the pooled analysis showed improvement in visual acuity, headaches, and papilledema in 36.5\%, $70 \%$, and $90 \%$ of reported ONSF cases, respectively. ${ }^{60}$ Interestingly, ONSF has been reported to improve headaches in up to $50 \%$ of patients and the mechanisms for such improvement are controversial. ${ }^{62,78,87}$ However, headaches can improve after CSF shunting to a greater degree than that reported for ONSF.

Many patients have sufficient visual improvement from unilateral surgery. Alsuhaibani et al reported that in 62 patients who underwent unilateral ONSF, the grade of papilledema significantly decreased in both ipsilateral (operated) and contralateral (unoperated) eyes. ${ }^{88}$ Thus, although bilateral ONSF might be necessary, many cases are improved in both eyes by a single surgery. Failure of the fellow eye to improve and the asymmetry of papilledema may be explained in part by the resistance to CSF flow produced by the trabeculations of the subarachnoid space or the tightness of the optic canal.

In general, we prefer ONSF for patients with predominantly visual symptoms who fail maximal medical therapy.

\section{Venous sinus stenting}

With the advent of improved endovascular technology, a number of case reports and case series have reported the use of dural venous sinus stent placement for the treatment of IIH ${ }^{89-92}$ Pooled analysis of patients with manometric and venographic evidence of focal venous stenosis demonstrated that stent placement improved visual symptoms, headaches, and papilledema $88 \%, 97 \%$, and $87 \%$ of the time, respectively. ${ }^{93}$ However, the interval between the procedure and improvement of symptoms was not addressed in the literature. Thus, the true efficacy of venous sinus stenting might be lower, knowing that IIH can be self-limited and resolves within months in some patients. ${ }^{94}$

The rationale for stenting is that lowering venous sinus pressure might lower ICP. It is unknown whether CVS stenosis is the cause or the sequel of IH in IIH. The use of computed tomographic venography and magnetic resonance venography in evaluation of IIH has led to the finding that many IIH patients (and 7\% of normal patients) have a non-thrombotic dural venous sinus stenosis of the transverse sinus. In a retrospective study of 51 patients with IIH and transverse sinus stenosis, $71 \%$ had more than $50 \%$ stenosis, but the degree and location of the stenosis did not predict the clinical outcome. ${ }^{95}$

Despite encouraging results, the major limitations of venous stenting include the requirement for dual antiplatelet therapy ( $\geq 90$ days) and potential life-threatening operative risks (eg, SDH and SAH). Further prospective studies are needed to evaluate the role of venous sinus stenting in the treatment of IIH.

\section{Summary}

The main mechanism of visual loss in papilledema is likely due to a translaminar elevation of the CSF pressure gradient that is transmitted down the optic nerve sheath from the intracranial cavity and produces axoplasmic flow stasis and resultant intraneuronal ischemia. Medical treatment with weight loss and diuretics (eg, acetazolamide) remains the mainstay of therapy for IIH, and surgical treatment is generally reserved for patients who fail, are intolerant to, or non-compliant with maximum medical therapy. Although most treated patients do not experience severe visual loss, some patients present with or develop a malignant, acute, or fulminant course that may require a more aggressive and urgent surgical treatment. Temporization with a lumbar drain while awaiting definitive surgical therapy (eg, ONSF, VPS/LPS), hospital admission, and aggressive intravenous medical therapy may be helpful..96,97 A much needed surgical IIH trial aiming to determine the optimal surgical management is currently underway. In the absence of an evidence-based treatment protocol, ONSF is our procedure of choice for papilledema-related visual loss, but CSF shunting procedures may be necessary, especially if intractable headache due to IH with or without visual loss is the predominant feature. The role of CVS stenting in IIH treatment has yet to be established, but early results are promising and a clinical trial is already underway. 


\section{Disclosure}

The authors report no conflicts of interest in this work.

\section{References}

1. Lee AG, Wall M. Papilledema: are we any nearer to a consensus on pathogenesis and treatment? Curr Neurol Neurosci Rep. 2012;12: 334-339.

2. Newman EW. Ocular signs of intracranial disease in children and juveniles. Am J Ophthalmol. 1938;21:286-292.

3. Durcan FJ, Corbett JJ, Wall M. The incidence of pseudotumor cerebri. Population studies in Iowa and Louisiana. Arch Neurol. 1988;45: 875-877.

4. Radhakrishnan K, Ahlskog JE, Cross SA, Kurland LT, O'Fallon WM. Idiopathic intracranial hypertension (pseudotumor cerebri). Descriptive epidemiology in Rochester, Minn, 1976 to 1990. Arch Neurol. 1993;50: 78-80.

5. Raoof N, Sharrack B, Pepper IM, Hickman SJ. The incidence and prevalence of idiopathic intracranial hypertension in Sheffield, UK. Eur J Neurol. 2011;18:1266-1268.

6. Almarzouqi SJ, Morgan ML, Lee AG. Idiopathic intracranial hypertension in the Middle East: a growing concern. Saudi J Ophthalmol. 2015;29:26-31.

7. Robertson DN, Dinsdale HB. The Nervous Sysytem. Baltimore, MD, USA: Williams \& Wilkins; 1972.

8. Sugerman HJ, DeMaria EJ, Felton WL, Nakatsuka M, Sismanis A. Increased intra-abdominal pressure and cardiac filling pressures in obesityassociated pseudotumor cerebri. Neurology. 1997;49:507-511.

9. Hayreh SS. Pathogenesis of optic disc oedema in raised intracranial pressure. Trans Ophthalmol Soc UK. 1976;96:404-407.

10. Hayreh SS. Optic disc edema in raised intracranial pressure. V Pathogenesis. Arch Ophthalmol. 1977;95:1553-1565.

11. Hayreh SS, March W, Anderson DR. Pathogenesis of block of rapid orthograde axonal transport by elevated intraocular pressure. Exp Eye Res. 1979;28:515-523.

12. Corbett JJ, Jacobson DM, Mauer RC, Thompson HS. Enlargement of the blind spot caused by papilledema. Am J Ophthalmol. 1988;105: 261-265.

13. Grehn F, Knorr-Held S, Kommerell G. Glaucomatouslike visual field defects in chronic papilledema. Albrecht Von Graefes Arch Klin Exp Ophthalmol. 1981;217:99-109.

14. Wall M, George D. Visual loss in pseudotumor cerebri. Incidence and defects related to visual field strategy. Arch Neurol. 1987;44: $170-175$.

15. Van Crevel H. Papilloedema, CSF pressure, and CSF flow in cerebral tumours. J Neurol Neurosurg Psychiatry. 1979;42:493-500.

16. Petrohelos MA, Henderson JW. The ocular findings of intracranial tumor. A study of 358 cases. Trans Am Acad Ophthalmol Otolaryngol. 1950;55:89-98.

17. Allen ED, Byrd SE, Darling CF, Tomita T, Wilczynski MA. The clinical and radiological evaluation of primary brain tumors in children. Part I: Clinical evaluation. J Natl Med Assoc. 1993;85:445-451.

18. Fahmy JA. Papilloedema associated with ruptured intracranial aneurysms. Acta Ophthalmol. 1972;50:793-802.

19. Columella F, Gaist G, Piazza G, Caraffa T. Extradural haematoma at the vertex. J Neurol Neurosurg Psychiatry. 1968;31:315-320.

20. Selhorst JB, Gudeman SK, Butterworth JF, Harbison JW, Miller JD, Becker DP. Papilledema after acute head injury. Neurosurgery. 1985;16: 357-363.

21. Hanna LS, Girgis NI, Yassin MW, et al. Incidence of papilloedema and optic atrophy in meningitis. Jpn J Ophthalmol. 1981;25:69-73.

22. Drake RL. Ocular syphilis. III. Review of the literature and report of a case of acute syphilitic meningitis and meningoencephalitis with special reference to papilledema. Arch Ophthalmol. 1933;9:234-243.

23. Lamonte M, Silberstein SD, Marcelis JF. Headache associated with aseptic meningitis. Headache. 1995;35:520-526.
24. Silverstein A. Papilledema with acute viral infections of the brain. Mt Sinai J Med. 1974;41:435-443.

25. Mittal MM, Gupta NC, Sharma ML. Spinal epidural meningioma associated with increased intracranial pressure. Neurology. 1970;20:818-820.

26. Schijman E, Zúccaro G, Monges JA. Spinal tumors and hydrocephalus. Childs Brain. 1981;8:401-405.

27. Mirone G, Cinalli G, Spennato P, Ruggiero C, Aliberti F. Hydrocephalus and spinal cord tumors: a review. Childs Nerv Syst. 2011;27: 1741-1749.

28. Gardner WJ, Spitler DK, Whitten C. Increased intracranial pressure caused by increased protein content in the cerebrospinal fluid; an explanation of papilledema in certain cases of small intracranial and intraspinal tumors, and in the Guillain-Barre syndrome. N Engl J Med. 1954;250:932-936.

29. Michowiz SD, Rappaport HZ, Shaked I, Yellin A, Sahar A. Thoracic disc herniation associated with papilledema. Case report. J Neurosurg. 1984;61:1132-1134.

30. Friedman DI, Jacobson DM. Diagnostic criteria for idiopathic intracranial hypertension. Neurology. 2002;59:1492-1495.

31. Dhungana S, Sharrack B, Woodroofe N. Idiopathic intracranial hypertension. Acta Neurol Scand. 2010;121:71-82.

32. Friedman DI. Medication-induced intracranial hypertension in dermatology. Am J Clin Dermatol. 2005;6:29-37.

33. Daniels AB, Liu GT, Volpe NJ, et al. Profiles of obesity, weight gain, and quality of life in idiopathic intracranial hypertension (pseudotumor cerebri). Am J Ophthalmol. 2007;143:635-641.

34. Rowe FJ, Sarkies NJ. The relationship between obesity and idiopathic intracranial hypertension. Int J Obes Relat Metab Disord. 1999;23: $54-59$.

35. Wall $\mathrm{M}$, George D. Idiopathic intracranial hypertension. A prospective study of 50 patients. Brain. 1991;114 Pt 1A:155-180.

36. Wall $\mathrm{M}$. The headache profile of idiopathic intracranial hypertension. Cephalalgia. 1990;10:331-335.

37. Thurtell MJ, Wall M. Idiopathic intracranial hypertension (pseudotumor cerebri): recognition, treatment, and ongoing management. Curr Treat Options Neurol. 2013;15:1-12.

38. Dill JL. Thrombosis of the sigmoid or lateral sinus. Arch Surg. 1934;29:705.

39. Bergui M, Bradac GB. Clinical picture of patients with cerebral venous thrombosis and patterns of dural sinus involvement. Cerebrovasc Dis. 2003;16:211-216.

40. Saposnik G, Barinagarrementeria F, Brown RD, et al. Diagnosis and management of cerebral venous thrombosis: a statement for healthcare professionals from the American Heart Association/American Stroke Association. Stroke. 2011;42:1158-1192.

41. Bertelsen TI. The premature synostosis of the cranial sutures. Acta Ophthalmol Suppl. 1958;36 Suppl 51:1-176.

42. Ciurea AV, Fountas KN, Coman TC, et al. Long-term surgical outcome in patients with intracranial hydatid cyst. Acta Neurochir (Wien). 2006;148: 421-426.

43. Desai KI, Nadkarni TD, Muzumdar DP, Goel AH. Surgical management of colloid cyst of the third ventricle - study of 105 cases. Surg Neurol. 2002;57:295-302.

44. Thambisetty M, Lavin PJ, Newman NJ, Biousse V. Fulminant idiopathic intracranial hypertension. Neurology. 2007;68:229-232.

45. Wong R, Madill SA, Pandey P, Riordan-Eva P. Idiopathic intracranial hypertension: the association between weight loss and the requirement for systemic treatment. BMC Ophthalmol. 2007;7:15.

46. Sinclair AJ, Burdon MA, Nightingale PG, et al. Low energy diet and intracranial pressure in women with idiopathic intracranial hypertension: prospective cohort study. BMJ. 2010;341:c2701.

47. Fridley J, Foroozan R, Sherman V, Brandt ML, Yoshor D. Bariatric surgery for the treatment of idiopathic intracranial hypertension. J Neurosurg. 2011;114:34-39.

48. Lee AG, Golnik K, Kardon R, Wall M, Eggenberger E, Yedavally S. Sleep apnea and intracranial hypertension in men. Ophthalmology. 2002;109:482-485. 
49. Jennum P, Børgesen SE. Intracranial pressure and obstructive sleep apnea. Chest. 1989;95:279-283.

50. Wall M, Purvin V. Idiopathic intracranial hypertension in men and the relationship to sleep apnea. Neurology. 2009;72:300-301.

51. Thurtell MJ, Bruce BB, Rye DB, Newman NJ, Biousse V. The Berlin questionnaire screens for obstructive sleep apnea in idiopathic intracranial hypertension. J Neuroophthalmol. 2011;31:316-319.

52. Wall M, Kupersmith MJ, Kieburtz KD, et al. The idiopathic intracranial hypertension treatment trial: clinical profile at baseline. JAMA Neurol. 2014;71:693-701.

53. Corbett JJ. The 1982 Silversides lecture. Problems in the diagnosis and treatment of pseudotumor cerebri. Can J Neurol Sci. 1983;10:221-229.

54. Celebisoy N, Gökçay F, Sirin H, Akyürekli O. Treatment of idiopathic intracranial hypertension: topiramate vs acetazolamide, an open-label study. Acta Neurol Scand. 2007;116:322-327.

55. Weisberg LA. Benign intracranial hypertension. Medicine (Baltimore). 1975;54:197-207.

56. Weisberg LA, Chutorian AM. Pseudotumor cerebri of childhood. Am J Dis Child. 1977;131:1243-1248.

57. Neville BG, Wilson J. Benign intracranial hypertension following corticosteroid withdrawal in childhood. Br Med J. 1970;3:554-556.

58. Liu GT, Kay MD, Bienfang DC, Schatz NJ. Pseudotumor cerebri associated with corticosteroid withdrawal in inflammatory bowel disease. Am J Ophthalmol. 1994;117:352-357.

59. Johnston I, Paterson A. Benign intracranial hypertension. II. CSF pressure and circulation. Brain. 1974;97:301-312.

60. Lai LT, Danesh-Meyer HV, Kaye AH. Visual outcomes and headache following interventions for idiopathic intracranial hypertension. J Clin Neurosci. 2014;21:1670-1678.

61. Mukherjee N, Bhatti MT. Update on the surgical management of idiopathic intracranial hypertension. Curr Neurol Neurosci Rep. 2014;14:438.

62. Spitze A, Malik A, Al-Zubidi N, Golnik K, Lee AG. Optic nerve sheath fenestration vs cerebrospinal diversion procedures. J Neuroophthalmol. 2013;33:183-188.

63. Feldon SE. Visual outcomes comparing surgical techniques for management of severe idiopathic intracranial hypertension. Neurosurg Focus. 2007;23:E6

64. Eggenberger ER, Miller NR, Vitale S. Lumboperitoneal shunt for the treatment of pseudotumor cerebri. Neurology. 1996;46:1524-1530.

65. Burgett RA, Purvin VA, Kawasaki A. Lumboperitoneal shunting for pseudotumor cerebri. Neurology. 1997;49:734-739.

66. Rosenberg ML, Corbett JJ, Smith C, et al. Cerebrospinal fluid diversion procedures in pseudotumor cerebri. Neurology. 1993;43:1071-1072.

67. Curry WT, Butler WE, Barker FG. Rapidly rising incidence of cerebrospinal fluid shunting procedures for idiopathic intracranial hypertension in the United States, 1988-2002. Neurosurgery. 2005;57:97-108.

68. Sinclair AJ, Kuruvath S, Sen D, Nightingale PG, Burdon MA, Flint G. Is cerebrospinal fluid shunting in idiopathic intracranial hypertension worthwhile? A 10-year review. Cephalalgia. 2011;31:1627-1633.

69. Malik A, Golnik K. Cerebrospinal fluid diversion procedures in the treatment of patients with idiopathic intracranial hypertension. Int Ophthalmol Clin. 2014;54:51-59.

70. Johnston I, Besser M, Morgan MK. Cerebrospinal fluid diversion in the treatment of benign intracranial hypertension. $J$ Neurosurg. 1988;69:195-202.

71. Abubaker K, Ali Z, Raza K, Bolger C, Rawluk D, O’Brien D. Idiopathic intracranial hypertension: lumboperitoneal shunts versus ventriculoperitoneal shunts - case series and literature review. Br J Neurosurg. 2011;25:94-99.

72. Tarnaris A, Toma AK, Watkins LD, Kitchen ND. Is there a difference in outcomes of patients with idiopathic intracranial hypertension with the choice of cerebrospinal fluid diversion site: a single centre experience. Clin Neurol Neurosurg. 2011;113:477-479.

73. Mcgirt MJ, Woodworth G, Thomas G, Miller N, Williams M, Rigamonti D. Cerebrospinal fluid shunt placement for pseudotumor cerebri-associated intractable headache: predictors of treatment response and an analysis of long-term outcomes. J Neurosurg. 2004;101:627-632.
74. Liu GT, Volpe NJ, Schatz NJ, Galetta SL, Farrar JT, Raps EC. Severe sudden visual loss caused by pseudotumor cerebri and lumboperitoneal shunt failure. Am J Ophthalmol. 1996;122:129-131.

75. El-Saadany WF, Farhoud A, Zidan I. Lumboperitoneal shunt for idiopathic intracranial hypertension: patients' selection and outcome. Neurosurg Rev. 2012;35:239-243.

76. Shaw GY, Million SK. Benign intracranial hypertension: a diagnostic dilemma. Case Rep Otolaryngol. 2012;2012:1-8.

77. Friedman DI, Jacobson DM. Idiopathic intracranial hypertension. J Neuroophthalmol. 2004;24:138-145.

78. Corbett JJ, Nerad JA, Tse DT, Anderson RL. Results of optic nerve sheath fenestration for pseudotumor cerebri. The lateral orbitotomy approach. Arch Ophthalmol. 1988;106:1391-1397.

79. Sergott RC, Savino PJ, Bosley TM. Optic nerve sheath decompression: a clinical review and proposed pathophysiologic mechanism. Aust NZ J Ophthalmol. 1990;18:365-373.

80. Spoor TC, Ramocki JM, Madion MP, Wilkinson MJ. Treatment of pseudotumor cerebri by primary and secondary optic nerve sheath decompression. Am J Ophthalmol. 1991;112:177-185.

81. Brourman ND, Spoor TC, Ramocki JM. Optic nerve sheath decompression for pseudotumor cerebri. Arch Ophthalmol. 1988;106:1378-1383.

82. Keltner JL. Optic nerve sheath decompression. How does it work? Has its time come? Arch Ophthalmol. 1988;106:1365-1369.

83. Acheson JF, Green WT, Sanders MD. Optic nerve sheath decompression for the treatment of visual failure in chronic raised intracranial pressure. J Neurol Neurosurg Psychiatry. 1994;57:1426-1429.

84. Hamed LM, Tse DT, Glaser JS, Byrne SF, Schatz NJ. Neuroimaging of the optic nerve after fenestration for management of pseudotumor cerebri. Arch Ophthalmol. 1992;110:636-639.

85. Smith CH, Orcutt JC. Surgical treatment of pseudotumor cerebri. Int Ophthalmol Clin. 1986;26:265-275.

86. Uretsky S. Surgical interventions for idiopathic intracranial hypertension. Curr Opin Ophthalmol. 2009;20:451-455.

87. Corbett JJ, Thompson HS. The rational management of idiopathic intracranial hypertension. Arch Neurol. 1989;46:1049-1051.

88. Alsuhaibani AH, Carter KD, Nerad JA, Lee AG. Effect of optic nerve sheath fenestration on papilledema of the operated and the contralateral nonoperated eyes in idiopathic intracranial hypertension. Ophthalmology. 2011;118:412-414.

89. Higgins JNP, Cousins C, Owler BK, Sarkies N, Pickard JD. Idiopathic intracranial hypertension: 12 cases treated by venous sinus stenting. J Neurol Neurosurg Psychiatry. 2003;74:1662-1666.

90. Arac A, Lee M, Steinberg GK, Marcellus M, Marks MP. Efficacy of endovascular stenting in dural venous sinus stenosis for the treatment of idiopathic intracranial hypertension. Neurosurg Focus. 2009;27:E14.

91. Donnet A, Metellus P, Levrier O, et al. Endovascular treatment of idiopathic intracranial hypertension: clinical and radiologic outcome of 10 consecutive patients. Neurology. 2008;70:641-647.

92. Bussière M, Falero R, Nicolle D, Proulx A, Patel V, Pelz D. Unilateral transverse sinus stenting of patients with idiopathic intracranial hypertension. AJNR Am J Neuroradiol. 2010;31:645-650.

93. Puffer RC, Mustafa W, Lanzino G. Venous sinus stenting for idiopathic intracranial hypertension: a review of the literature. J Neurointerv Surg. 2013;5:483-486.

94. Bruce BB, Biousse V, Newman NJ. Update on idiopathic intracranial hypertension. Am J Ophthalmol. 2011;152:163-169.

95. Riggeal BD, Bruce BB, Saindane AM, et al. Clinical course of idiopathic intracranial hypertension with transverse sinus stenosis. Neurology. 2013;80:289-295

96. Shah VA, Kardon RH, Lee AG, Corbett JJ, Wall M. Long-term follow-up of idiopathic intracranial hypertension: the Iowa experience. Neurology. 2008;70:634-640.

97. Wall M. Idiopathic intracranial hypertension. Neurol Clin. 2010;28: 593-617. 
Eye and Brain

\section{Publish your work in this journal}

Eye and Brain is an international, peer-reviewed, open access journal focusing on clinical and experimental research in the field of neuroophthalmology. All aspects of patient care are addressed within the journal as well as basic research. Papers covering original research, basic science, clinical and epidemiological studies, reviews and evaluations,

Submit your manuscript here: http://www.dovepress.com/eye-and-brain-journal

\section{Dovepress}

guidelines, expert opinion and commentary, case reports and extended reports are welcome. The manuscript management system is completely online and includes a very quick and fair peer-review system, which is all easy to use. Visit http://www.dovepress.com/testimonials.php to read real quotes from published authors. 